\title{
Tetrahydrocannabinol and Skin Cancer: Analysis of YouTube Videos
}

Andrina Mamo ${ }^{1}$, BS; Mindy D Szeto ${ }^{1}, \mathrm{MS}$; Roya Mirhossaini ${ }^{2}, \mathrm{MD}$; Andrew Fortugno ${ }^{3}, \mathrm{MD}$; Robert P Dellavalle ${ }^{1}$, $\mathrm{MD}, \mathrm{PhD}, \mathrm{MSPH}$

\footnotetext{
${ }^{1}$ Department of Dermatology, University of Colorado Anschutz Medical Campus, Aurora, CO, United States

${ }^{2}$ Department of Medicine, Riverside Community Hospital, Riverside, CA, United States

${ }^{3}$ Department of Medicine, University of Tennessee Health Sciences Center, Memphis, TN, United States
}

\author{
Corresponding Author: \\ Mindy D Szeto, MS \\ Department of Dermatology \\ University of Colorado Anschutz Medical Campus \\ 1665 Aurora Court, 3rd Floor \\ Mailstop F703 \\ Aurora, CO, 80045 \\ United States \\ Phone: 17208480500 \\ Email: mindy.szeto@cuanschutz.edu
}

\begin{abstract}
Background: Cannabis oil is being used topically by patients with skin cancer as a homeopathic remedy, and has been promoted and popularized on social media, including YouTube. Although topical cannabinoids, especially tetrahydrocannabinol (THC), may have antitumor effects, results from a sparse number of clinical trials and peer-reviewed studies detailing safety and efficacy are still under investigation.
\end{abstract}

Objective: We sought to assess the accuracy, quality, and reliability of THC oil and skin cancer information available on YouTube.

Methods: The 10 most-viewed videos on THC oil and skin cancer were analyzed with the Global Quality Scale (GQS), DISCERN score, and useful/misleading criteria based on presentation of erroneous and scientifically unproven information. The videos were also inspected for source, length, and audience likes/dislikes. Top comments were additionally examined based on whether they were favorable, unfavorable, or neutral regarding the video content.

Results: All analyzed videos $(10 / 10,100 \%)$ received a GQS score of 1 , corresponding to poor quality of content, and 9/10 $(90 \%)$ videos received a DISCERN score of 0 , indicating poor reliability of information presented. All 10 videos were also found to be misleading and not useful according to established criteria. Top comments were largely either favorable (13/27, $48 \%)$ or neutral $(13 / 27,48 \%)$ toward the content of the videos, compared to unfavorable $(1 / 27,4 \%)$.

Conclusions: Dermatologists should be aware that the spread of inaccurate information on skin cancer treatment currently exists on popular social media platforms and may lead to detrimental consequences for patients interested in pursuing alternative or homeopathic approaches.

(JMIR Dermatol 2021;4(1):e26564) doi: $10.2196 / 26564$

\section{KEYWORDS}

THC; tetrahydrocannabinol; skin cancer; YouTube; cannabis; social media; internet

\section{Introduction}

Cannabis oil as a homeopathic remedy for skin cancer was most popularized by Rick Simpson, a Canadian medical marijuana activist. In 2003, Rick Simpson claimed that he was able to cure his basal cell carcinoma with his specially extracted "Rick
Simpson Oil," an illegally produced high-tetrahydrocannabinol (THC) oil made from Cannabis indica [1]. An online documentary called Healing Cancer with Cannabis: The Rick Simpson Story [2] currently has over 150,000 views on YouTube and documents Rick Simpson's journey to curing both his skin cancer and that of others, including basal cell 
carcinoma and melanoma. Given the increasing importance of social media and YouTube in disseminating information about health care and dermatology [3], we sought to characterize the quality of information patients attain from popular YouTube videos concerning THC and skin cancer.

\section{Methods}

On June 5, 2020, we searched YouTube using the phrase "THC skin cancer." The ranking option of "view count" was selected. The search resulted in a total of 32 videos; however, only nonduplicate videos with over 1000 views were analyzed in order to obtain accurate representation and capture the most popular videos that had reached the largest YouTube audiences. Two independent reviewers viewed and evaluated all videos, and any discrepancies between reviewers were discussed and resolved in a consensus meeting. All reviewers were experienced in skin cancer pathogenesis, clinical presentation, and treatment. Various predetermined attributes were surveyed, and videos were scored for quality and usefulness with the 5-point Likert scale Global Quality Scale (GQS) as described in Textbox 1 $[3,4]$.

Textbox 1. Global Quality Scale 5-point scale description.

1. Poor quality, poor flow of the video, most information missing, not at all useful for patients. I would highly discourage a patient with skin cancer from watching this video.

2. Generally poor quality and poor flow. Some information listed but many important topics missing. Of very limited use to patients. I would discourage a patient with skin cancer from watching this video.

3. Moderate quality, suboptimal flow. Some important information is adequately discussed but other information is poorly discussed. Somewhat useful for patients. I would neither encourage nor discourage a patient with skin cancer from watching this video.

4. Good quality and generally good flow. Most of the relevant information is listed, but some topics not covered. Useful for patients. I would encourage a patient with skin cancer to watch this video.

5. Excellent quality and flow, very useful for patients. I would highly encourage a patient with skin cancer to watch this video.

Videos were additionally rated on an adapted DISCERN 5-point reliability scale, an assessment of health information quality used in previous studies [3,5]. Scoring of content accounted for the breadth of skin cancer information discussed, including epidemiology, pathogenesis, clinical features, and treatment. One DISCERN point was earned for each criteria fulfilled, for a maximum of 5 points (Textbox 2).

Textbox 2. Adapted DISCERN content reliability score description.

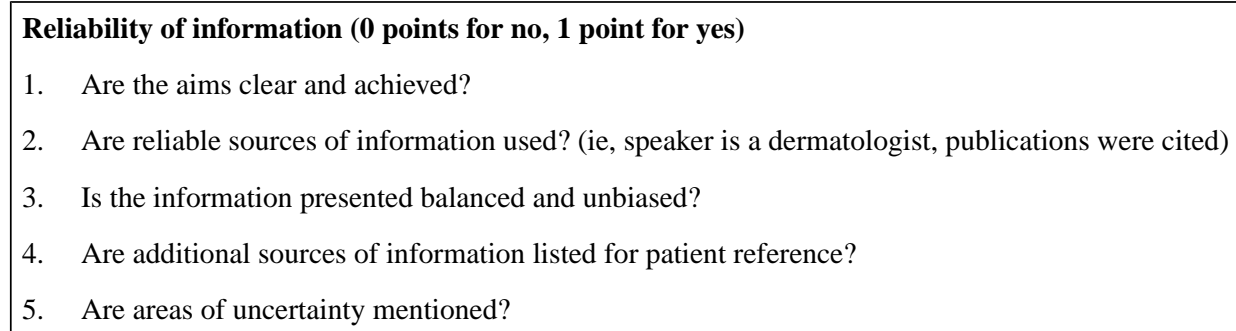

Videos were further classified as useful or misleading based on the following yes/no criteria established in prior literature [6-10]:

1. Useful, if they contained scientifically sound information about any aspect of skin cancer.

2. Misleading, if they contained scientifically erroneous or unproven information about any aspect of skin cancer.

For each video, the top 3 comments determined by YouTube according to the number of "thumbs up" ratings were additionally assessed for whether the comment was favorable, neutral, or unfavorable toward the video content. The source and date of the comment were also recorded.

\section{Results}

The 10 videos surveyed (Multimedia Appendix 1) had a total view count of 645,821 views, with an average of 64,582 views per video. Video length ranged from around 2 minutes to over 107 minutes. Sources of videos were varied, and included cannabis companies, Rick Simpson affiliates, and patient perspectives. The surveyed videos had positive social engagement, with a cumulative "thumbs up" score of 4923, and a "thumbs down" score of 183 .

Overall, 10/10 videos (100\%) had a GQS score of 1, corresponding to "poor quality, poor flow of the video, most information missing, not at all useful for patients." Just 1 of 10 $(10 \%)$ videos received a score of 1 of a possible 5 points on the DISCERN scale, corresponding to poor content reliability, while $9 / 10(90 \%)$ videos received a score of 0 . All 10 videos received a "no" rating according to the useful criteria, and a "yes" rating for misleading.

Summaries of video content and top comments for each video are shown in Multimedia Appendix 2. The commenting feature was turned off for one video. Overall, comments were all posted by individual YouTube users, and were largely favorable or neutral toward the video content, with 13/27 comments (48\%) 
classified as favorable, 13/27 (48\%) neutral, and 1/27 (4\%) unfavorable.

\section{Discussion}

\section{Principal Findings}

Patients are increasingly interested in and selecting nontraditional and alternative therapies for a variety of health conditions. Cannabis oil has achieved great popularity in the past few decades for the treatment of both nonmelanoma and melanoma skin cancers, despite the current lack of evidence; no clinical trials have yet to test their safety and efficacy in humans. Although early preclinical studies have shown that cannabinoids and cannabinoid derivatives may potentially have antitumor effects on keratinocyte carcinoma and melanoma [11], other studies have demonstrated that cannabinoids can be potent proinflammatory chemotactic agents in cell culture models [12].

The body's endocannabinoid system regulates cell growth through primary endocannabinoids, such as arachidonoyl ethanolamide (AEA) and 2-arachidonoyl glycerol (2-AG), and their metabolism by fatty acid amide hydrolase (FAAH). As detailed in previous reports [13], cannabinoid receptors CB1 and CB2 are found to be expressed on both nonmelanoma and melanoma skin cancers, with the former being largely expressed in the synaptic terminal in order to regulate neurotransmission, and the latter playing a role in activation of psychoactive properties [13].

Melanoma has largely been attributed to chronic sun damage (CSD) as well as non-CSD causes due to mutations in the cell regulatory pathway [14]. A study conducted by Armstrong et al [15] on melanoma cells treated with the cannabinoid THC displayed antitumor properties through the activation of autophagy and apoptotic pathways in vivo and in vitro. Similarly, cannabinoids have displayed antitumor effects in several other studies, focusing on CB2's anti-inflammatory properties and inhibition of Akt, a key element in the survival pathway of melanomas [16,17].

Cannabinoids have also demonstrated antitumor effects in nonmelanoma skin cancers. Squamous cell carcinomas (SCCs) are some of the most common cancers in humans and have been linked to risk factors including, but not limited to, UV exposure, chemical carcinogens, and viral infections [18]. Induction of apoptosis and tumor regression as an established effect of cannabinoid application has become evident through depression of angiogenic factors, such as vascular endothelial growth factor (VEGF) and angiopoietin-2 (Ang2), as well as decreasing activation of epidermal growth factor receptors (EGF-R) [19].

Although promising research on the treatment of skin cancers with cannabinoids is currently being conducted, the spread of information rooted in evidence-based medicine remains minimal on social media sites such as YouTube. Analysis of video content by two separate reviewers with health care experience and an educational background in skin cancer resulted in assessment of the information presented as uniformly misleading to viewers, along with a GQS of 1 assigned to all videos, demonstrating the pervasiveness of poor-quality information, and also largely unreliable content according to DISCERN criteria. It is concerning that top comments in response to these videos were overwhelmingly either favorable or neutral, highlighting the possibility that fake or secondary individual user accounts could be commenting to generate the outward appearance of validity and support for the video content. With the increasing use of social media, viral content, and thus the immense audience that can be reached via different platforms, including YouTube, inaccurate information can easily be spread to viewers who may be searching for solutions to skin-related problems. It is important for dermatologists to be aware that social media use may subsequently encourage patients to rely on information not provided by trained physicians and health care teams. Potentially harmful side effects or adverse consequences could be experienced by patients due to the dissemination of incorrect or poorly understood information. An increase in the presence of board-certified dermatologists on social media platforms would allow for improved patient education and propagation of medically accurate information to audiences seeking knowledge on skin cancer treatment with cannabinoids.

\section{Conclusion}

This study reiterates the importance of accessible, trustworthy, and engaging educational content curated by medical professionals for patients seeking information about skin cancer treatment online. In the surveyed YouTube data, no videos were curated by medical professionals tackling the popular issue of THC for the treatment of skin cancer, thus highlighting an opportunity for future engagement on social media to improve health education.

\section{Conflicts of Interest}

RPD is a Joint Coordinating Editor for Cochrane Skin, a dermatology section editor for UpToDate, a Social Media Editor for the Journal of the American Academy of Dermatology (JAAD), and a Podcast Editor for the Journal of Investigative Dermatology (JID). He is a coordinating editor representative on Cochrane Council. RPD receives editorial stipends (JAAD, JID), royalties (UpToDate), and expense reimbursement from Cochrane Skin. RPD is the Editor-in-Chief for JMIR Publications since April 2021, but had no role in reviewing this paper for publication in JMIR Dermatology. The other authors report no conflicts of interest.

\section{Multimedia Appendix 1}

Attributes of evaluated YouTube videos.

[DOCX File, 17 KB-Multimedia Appendix 1] 


\section{Multimedia Appendix 2}

Content summary and evaluation of the top 3 comments posted for each YouTube video.

[DOCX File, 17 KB-Multimedia Appendix 2]

\section{References}

1. Li JY, Kampp JT. Review of Common Alternative Herbal "Remedies" for Skin Cancer. Dermatol Surg 2019;45(1):58-67. [doi: $10.1097 /$ dss.0000000000001622]

2. Harrigan C. Healing Cancer with Cannabis: The Rick Simpson Story. 2017 May 12. URL: https://www.youtube.com/ watch?v=Ak3Zo7-hNO0) [accessed 2020-09-21]

3. Qi J, Trang T, Doong J, Kang S, Chien A. Misinformation is prevalent in psoriasis-related YouTube videos. Dermatol Online J 2016 Nov 15;22(11):13030/qt7qc9z2m5. [Medline: 28329562]

4. Bernard A, Langille M, Hughes S, Rose C, Leddin D, Veldhuyzen van Zanten S. A systematic review of patient inflammatory bowel disease information resources on the World Wide Web. Am J Gastroenterol 2007 Sep;102(9):2070-2077. [doi: 10.1111/j.1572-0241.2007.01325.x] [Medline: 17511753 ]

5. Charnock D, Shepperd S, Needham G, Gann R. DISCERN: an instrument for judging the quality of written consumer health information on treatment choices. J Epidemiol Community Health 1999 Feb 01;53(2):105-111 [FREE Full text] [doi: 10.1136/jech.53.2.105] [Medline: $\underline{10396471]}$

6. Pandey A, Patni N, Singh M, Sood A, Singh G. YouTube as a source of information on the H1N1 influenza pandemic. Am J Prev Med 2010 Mar;38(3):e1-e3. [doi: 10.1016/j.amepre.2009.11.007] [Medline: 20171526]

7. Murugiah K, Vallakati A, Rajput K, Sood A, Challa NR. YouTube as a source of information on cardiopulmonary resuscitation. Resuscitation 2011 Mar;82(3):332-334. [doi: 10.1016/j.resuscitation.2010.11.015] [Medline: 21185643]

8. Singh AG, Singh S, Singh PP. YouTube for information on rheumatoid arthritis--a wakeup call? J Rheumatol 2012 May 01;39(5):899-903. [doi: 10.3899/jrheum.111114] [Medline: 22467934]

9. Sood A, Sarangi S, Pandey A, Murugiah K. YouTube as a source of information on kidney stone disease. Urology 2011 Mar;77(3):558-562. [doi: 10.1016/j.urology.2010.07.536] [Medline: 21131027]

10. Delli K, Livas C, Vissink A, Spijkervet F. Is YouTube useful as a source of information for Sjögren's syndrome? Oral Dis 2016 Apr 11;22(3):196-201. [doi: 10.1111/odi.12404] [Medline: 26602325]

11. Mounessa JS, Siegel JA, Dunnick CA, Dellavalle RP. The role of cannabinoids in dermatology. J Am Acad Dermatol 2017 Jul;77(1):188-190. [doi: 10.1016/j.jaad.2017.02.056] [Medline: 28416341]

12. Klein TW. Cannabinoid-based drugs as anti-inflammatory therapeutics. Nat Rev Immunol 2005 May;5(5):400-411. [doi: 10.1038/nri1602] [Medline: 15864274]

13. Scheau C, Badarau IA, Mihai L, Scheau A, Costache DO, Constantin C, et al. Cannabinoids in the Pathophysiology of Skin Inflammation. Molecules 2020 Feb 04;25(3):652 [FREE Full text] [doi: 10.3390/molecules25030652] [Medline: 32033005]

14. Turner N, Ware O, Bosenberg M. Genetics of metastasis: melanoma and other cancers. Clin Exp Metastasis 2018 Aug 2;35(5-6):379-391. [doi: 10.1007/s10585-018-9893-y] [Medline: 29722002]

15. Armstrong JL, Hill DS, McKee CS, Hernandez-Tiedra S, Lorente M, Lopez-Valero I, et al. Exploiting cannabinoid-induced cytotoxic autophagy to drive melanoma cell death. J Invest Dermatol 2015 Jun;135(6):1629-1637 [FREE Full text] [doi: 10.1038/jid.2015.45] [Medline: 25674907]

16. Kis B, Ifrim FC, Buda V, Avram S, Pavel IZ, Antal D, et al. Cannabidiol-from Plant to Human Body: A Promising Bioactive Molecule with Multi-Target Effects in Cancer. Int J Mol Sci 2019 Nov 25;20(23):5905 [FREE Full text] [doi: 10.3390/ijms20235905] [Medline: 31775230]

17. Blázquez C, Carracedo A, Barrado L, Real PJ, Fernández-Luna JL, Velasco G, et al. Cannabinoid receptors as novel targets for the treatment of melanoma. FASEB J 2006 Dec 25;20(14):2633-2635. [doi: 10.1096/fj.06-6638fje] [Medline: 17065222]

18. Dotto G, Rustgi A. Squamous Cell Cancers: A Unified Perspective on Biology and Genetics. Cancer Cell 2016 May 09;29(5):622-637 [FREE Full text] [doi: 10.1016/j.ccell.2016.04.004] [Medline: 27165741]

19. Casanova ML, Blázquez C, Martínez-Palacio J, Villanueva C, Fernández-Aceñero MJ, Huffman JW, et al. Inhibition of skin tumor growth and angiogenesis in vivo by activation of cannabinoid receptors. J Clin Invest 2003 Jan 1;111(1):43-50. [doi: 10.1172/jci200316116]

\section{Abbreviations}

2-AG: 2-arachidonoyl glycerol

AEA: arachidonoyl ethanolamide

AP-2: angiopoietin-2

CSD: chronic sun damage

EGF-R: epidermal growth factor receptor

FAAH: fatty acid amide hydrolase

GQS: Global Quality Scale

SCC: squamous cell carcinoma 
THC: tetrahydrocannabinol

VEGF: vascular endothelial growth factor

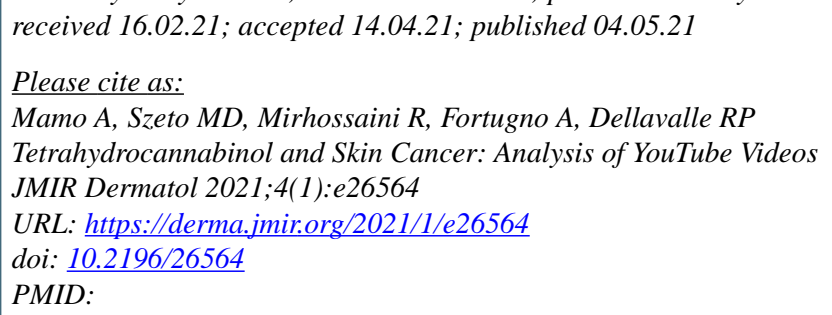

(CAndrina Mamo, Mindy D Szeto, Roya Mirhossaini, Andrew Fortugno, Robert P Dellavalle. Originally published in JMIR Dermatology (http://derma.jmir.org), 04.05.2021. This is an open-access article distributed under the terms of the Creative Commons Attribution License (https://creativecommons.org/licenses/by/4.0/), which permits unrestricted use, distribution, and reproduction in any medium, provided the original work, first published in JMIR Dermatology Research, is properly cited. The complete bibliographic information, a link to the original publication on http://derma.jmir.org, as well as this copyright and license information must be included. 